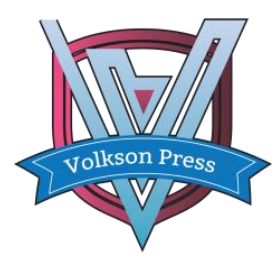

Contents List available at VOLKSON PRESS

Mechanical and Control Engineering (MCE)

DOI : http://doi.org/10.26480/wsmce.01.2017.06.08

\title{
COMPARATIVE STUDY ON DEFORMATION CHARACTERISTICS OF AL SINGLE CRYSTAL UNDER DYNAMIC AND QUASI-STATIC COMPRESSION
}

\author{
Meng Jiahong1*，Li Shukui1,2,3，Liu Jinxu1,2, Ma Siming1 \\ ${ }^{1}$ School of Material Science and Engineering, Beijing Institute of Technology (BIT), No.5 Zhongguancun South Street, Beijing, 100081, P.R. China \\ ${ }^{2}$ Nationa Key Laboratory of Science and Technology on Materials under shock and impact, Beijing, P.R. China \\ ${ }^{3}$ State Key Laboratory of Explosion Science and Technology, Beijing, P.R. China. \\ *Corresponding Author email: mengjiahong7@163.com
}

This is an open access article distributed under the Creative Commons Attribution License, which permits unrestricted use, distribution, and reproduction in any medium, provided the original work is properly cited

\section{ARTICLE DETAILS}

\section{Article History:}

Received 02 october 2017 Accepted 06 october 2017 Available online 11 november 2017

Keywords:

single crystal aluminum, dynamic and quasi-static, slip band, dislocation

\section{ABSTRACT}

The aluminum single crystal with face-centered cubic (FCC) structure was subjected to quasi-static and dynamic uniaxial compressive loading along the direction, respectively. The surface appearance, morphology and orientation of the loaded samples were analyzed. The experimental results showed that the deformation mechanisms of single crystal aluminum were both dislocations under quasi-static and dynamic loading, and the samples underwent non-uniform plastic deformation from single crystal to polycrystal. The causes of the nonuniform plastic deformation are as follows: the two sets of slip systems actuated by single-crystal aluminum under quasi-static loading have obvious sequential order and different slip amount; the material deforms rapidly under dynamic loading, and the distribution of slip band has obvious local concentration. The microstructure evolution of the aluminum single crystal into polycrystal is as follows: First, the thick strip-shaped deformation structure is formed by the dislocation slip, and as the deformation amount increases, the quasi-static sample forms a subgrain boundary by the internal dislocation accumulation, while the dynamic sample forms a subgrain boundary by dislocation rotation. Then, the unstable subgrain boundary is gradually transformed into a low angle grain boundary during the deformation process, later forming a high angle grain boundary.

\section{Introduction}

Crystal plastic deformation has always been a hot research topic in the field of materials. Crystal plastic theory can reveal the deformation law of crystal materials in depth, which means important theoretical value for the design, preparation and engineering application of guiding materials. Discussion on plastic deformation behavior of polycrystalline materials is an arduous and complex project, as the deformation of grains in polycrystalline materials is not uniform. In order to study the plastic deformation behavior of polycrystalline materials, the behavior of individual grains needs to be studied. The study on the mechanical behavior and the micro-deformation mechanism of single-crystalline materials in the deformation process can well explain their behavior [1]. Therefore, the research on the mechanism of plastic deformation of single crystal is the basis and guide to discuss the plastic deformation behavior of polycrystalline materials and even complex multiphase materials.

At present, abundant researches on the deformation mechanism of single crystal materials under quasi-static loading has been carried out one after another [2-4]. However, insufficient research has been done on the deformation mechanism of single crystal materials under dynamic loading. A studied the microstructure and deformation mechanism of close-packed hexagonal (HCP) magnesium single crystals under quasistatic and high strain rate compressive loads [5]. The results showed that the samples under static loading became polycrystal with twin crystal appeared in the samples, while the samples under dynamic loading with high strain rate remained single crystal, indicating great difference in mechanical behavior of the material under dynamic loading and quasistatic loading.

Single crystal aluminum is a typical face-centered cubic metal, showing obvious non-uniform macroscopic plastic deformation during loading deformation. Up to now, relevant researches have been carried out on the microscopic deformation process of single crystal aluminum. A studied the fatigue deformation behavior of aluminum single crystal [6]. The results showed that the slip band produced by Al single crystal was characterized by wave slip. The most typical dislocation distribution was cellular structure. The density of dislocations was relatively low in the unit cell center, with most dislocations concentrated in the cell wall. The researchers analyzed the deformation behavior from the perspective of dislocation, but did not reveal the specific structural change process.

In summary, the research on the deformation mechanism of single crystal aluminum under quasi-static and dynamic loading has the following problems: 1. few studies have been carried out on the deformation mechanism of single crystal aluminum under dynamic loading; 2 . the reason for the plastic deformation of single crystal aluminum remains unclear. 3. Insufficient study is done on structural evolution process of single crystal aluminum after loading deformation. In view of the above problems, cubic single-crystal aluminum material was subjected to uniaxial compressive loading under quasi-static and dynamic conditions to reveal the deformation mechanism of macroscopic plastic deformation of aluminum single crystal and microstructure evolution process from single crystal to polycrystal, as well as differences in deformation characteristics of aluminum single crystals under quasi-static and dynamic loading from the aspects of surface appearance, morphology and orientation characteristics.

\section{EXPERIMENT}

Single crystal aluminum samples were subjected to uniaxial dynamic and uniaxial quasi-static compressive loading, respectively. By analyzing the surface appearance, morphology and orientation characteristics of the deformed samples, the plastic deformation mechanism and microstructure evolution of single crystal aluminum under uniaxial compressive loading were studied.

Single crystal aluminum samples were loaded in the [11-1] direction with surfaces (112) and (1-10) observed, respectively, and the sample size was $5 \mathrm{~mm} \times 5 \mathrm{~mm} \times 5 \mathrm{~mm}$. After surface treatment of the two observation surfaces of the single crystal aluminum sample, the sample surface was detected by using EBSD to determine whether the sample was single crystal and whether the crystal plane indices of each surface of the single crystal aluminum sample was obtained. Then, the single crystal aluminum was subjected to uniaxial compressive loading in the direction of [11-1], the compression strain was controlled to 0.3 with the spacing ring, and the quasi-static loading with a strain rate of $1 \times 10^{-3} \mathrm{~s}^{-1}$ was carried out by 
electronic universal testing machine, and dynamic loading with a strain rate of $3 \times 10^{3} \mathrm{~s}^{-1}$ was carried out by Split Hopkinson Bar (SHPB).

The deformed sample surface was directly observed by Hitachi S-4800 cold field emission scanning electron microscope to analyze macroscopic surface features and slip band distribution characteristics and then study dislocation slip plastic deformation mechanism of single crystal aluminum under uniaxial compressive loading. The deformed sample was detected by EBSD, the microstructure morphology and orientation information characteristics of the sample after plastic deformation were analyzed to study the transformation mechanism of single crystal aluminum from single crystal to polycrystal under uniaxial compressive loading.

\section{RESULTS AND DISCUSSION}

Before loading experiments, the crystal plane indices of three planes perpendicular to each other on the original single-crystal aluminum samples were calibrated by the EBSD test technique to determine if the sample was single crystal. The results of the EBSD test are shown in Figure 1. Figure $1 \mathrm{a}$ is the EBSD orientation imaging of the aluminum single crystal sample and Fig. $1 \mathrm{~b}$ is the EBSD reverse polarity image of the aluminum single crystal sample. The figure data collection rate is close to $100 \%$. As can be seen from Figure 1a, the observed surfaces have the same orientation, indicating that the sample is close to the ideal single crystal. It can be confirmed from Figure $1 \mathrm{~b}$ that the three outer surfaces of the sample are (1-10), (11-1) and (112), respectively.
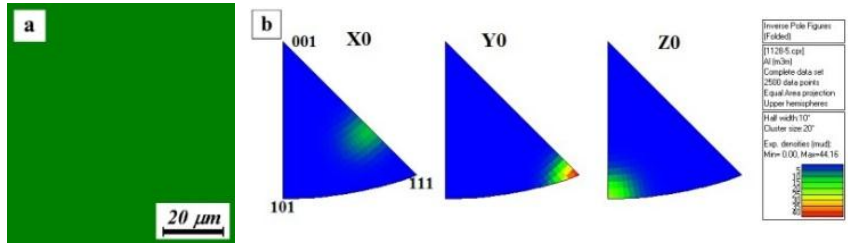

Figure 1: EBSD results of the original aluminum single crystal sample: (a) Orientation image (b) Reverse polarity image

After quasi-static and dynamic loading, the aluminum single crystal samples in cube before loading were deformed into irregular geometric polyhedrons. Figure 2 shows the macroscopic morphology of the deformed sample surface. Figure 2a shows the surface morphology of plane (1-10) after quasi-static compression. Figure $2 b$ shows the surface morphology of plane (112) after quasi-static compression. Figure 2c shows the surface morphology of plane (1-10) after dynamic compression. Figure $2 \mathrm{~d}$ shows the surface morphology of plane (112) after dynamic compression. From the figure, it can be seen that obvious macrofluctuations are shown on the deformed sample surface, mainly including deformed strips, protrusions, depressions and wrinkles seen from morphology. These macro-fluctuations are distributed in the local area of the sample surface, of which, the wrinkles are mainly distributed in the edge region of the sample surface, indicating that, under the dynamic and quasi-static compressive loading conditions, the macro-deformation of the aluminum single crystal sample exhibits inhomogeneous deformation characteristics. Surface undulations of the aluminum single crystal sample are more pronounced with more folds under the dynamic loading condition, which indicates a more inhomogeneous macroscopic deformation of the dynamically loaded sample compared to a quasistatically loaded one.

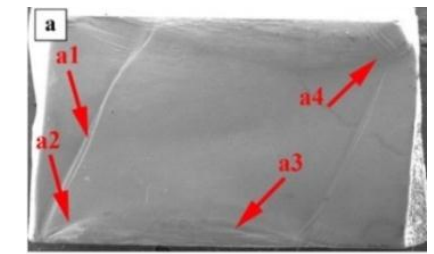

$1 \mathrm{~mm}$

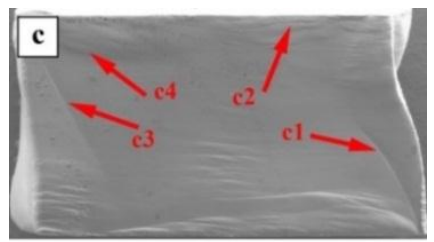

$1 \mathrm{~mm}$
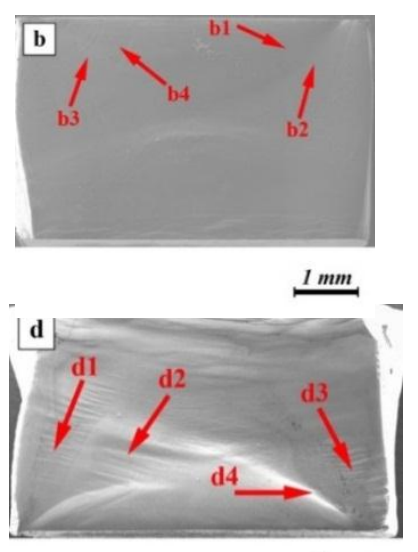

$1 \mathrm{~mm}$

Figure 2: Macroscopic morphology of the sample surface after

compression: (a) surface morphology of plane (1-10) after quasi-static compression; (b) surface morphology of plane (112) after quasi-static compression; (c) surface morphology of plane (1 -10) after dynamic compression; (d) surface morphology of plane (112) after dynamic compression

In order to reveal the reason for non-uniform plastic deformation, highmagnification observation was made on the area with obvious fluctuation. The microstructure characteristics were analyzed to study dislocation slip-based plastic deformation process of single crystal aluminum. The results of SEM observation are shown in Figure 3 and Figure 4. Figure 3 shows the micro-morphology of the surface after quasi-static loading of single crystal aluminum, and Figure 4 shows the micro-morphology of the surface after dynamic loading of single crystal aluminum. By observing the distribution characteristics of the slip band on the sample surface, it can be found that slip band is mainly distributed in the following modes: (1) slip band in single group parallel arrangement with equal spacing (such as a1, a3, b4, c2, (b1, c1, c3, c4, d1, d2, d3); (2) cluster slip band in single group parallel arrangement with unequal spacing (b1, c1, c3); (3) nonuniformly distributed cross-slip band (a2, a4, b2, b3); (4) uniformly distributed cross-slip band(d4).

The micro-topography of the sample surface after quasi-static compressive loading of single-crystal aluminum was analyzed. As can be seen from Figure 3, the average distance between the slip bands is about $4 \mu \mathrm{m}$. In local areas with larger macroscopic deformation, crossing of two groups of slip bands is shown. By observing the slip band with crossing features, obvious sequential order can be found in the two slip actuations. In some areas, it is obviously observed that the post-actuated slip system passes through the pre-actuated one, causing the previously formed slip band to be significantly bent, no longer flat and continuous, resulting in a stepped shape.

Therefore, under the quasi-static loading, the non-uniform plastic deformation process of the single crystal is as follows: due to the slow quasi-static loading process, during the loading, the deformed area first actuates a certain slip system to perform directional plastic deformation by dislocation slip. With the increasing deformation amount, the dislocations continuously accumulate, leading to internal stress concentration, so that another set of slip system is actuated. Then, through the dislocation slip of the set of slip system, the area performs directional plastic deformation. The successively actuated slip systems have different slip directions, and the slip amount is also very likely different. Therefore, the successively actuated slip deformations with different slip ratios result in inhomogeneous macroscopic plastic deformation, and single crystal shape appears as an irregular geometric polyhedron after deformation.

Figure 3: The surface micro-morphology of the single crystal aluminum after quasi-static loading: (1) a1, a2, a3 and a4 are the micro-morphology
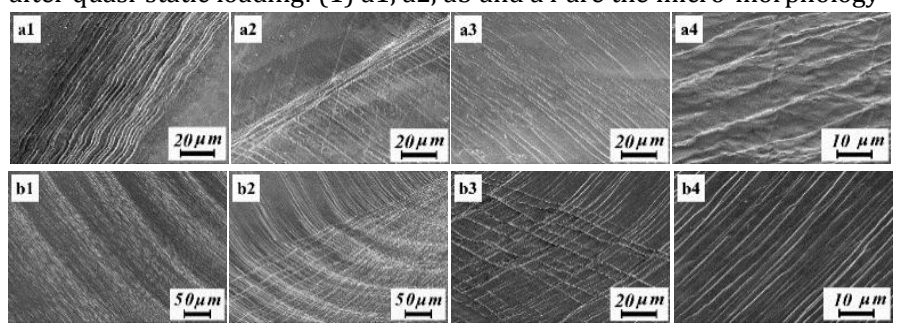

of plane (1-10) after quasi-static loading of single crystal aluminum, respectively corresponding to the micrographs of the area indicated by the arrows in Figure 2a; (2) b1, b2, b3 and b4 are micro-morphology of plane (112) after quasi-static compression of single crystal aluminum, respectively corresponding to the micrographs of the area indicated by the arrows in Figure 2b

The microcosmic morphology of the sample surface after dynamic compression loading of single crystal aluminum was analyzed. It can be seen from Figure 4 that the average distance between the slip bands is about $1 \mu \mathrm{m}$. More cross-slip is observed on the (112) plane, but there was no obvious sequential order in the actuation of the two slip systems, and the resulting grids are uniformly distributed. Compared with the sample after quasi-static loading, sample under dynamic loading has larger macro-deformation, straighter and denser slip band, and the distribution area of slip band is more concentrated.

Therefore, the non-uniform plastic deformation of the single crystal under dynamic loading is as follows: during the loading process, the material deforms rapidly, and a large number of slip systems are actuated almost simultaneously. Dislocations only need a small displacement along each set of slip systems to perform complete deformation, forming a flat and intensive slip band. However, plastic deformation of the material has very 
obvious local concentration, so single crystal also appears as irregular geometric polyhedron after dynamic loading.

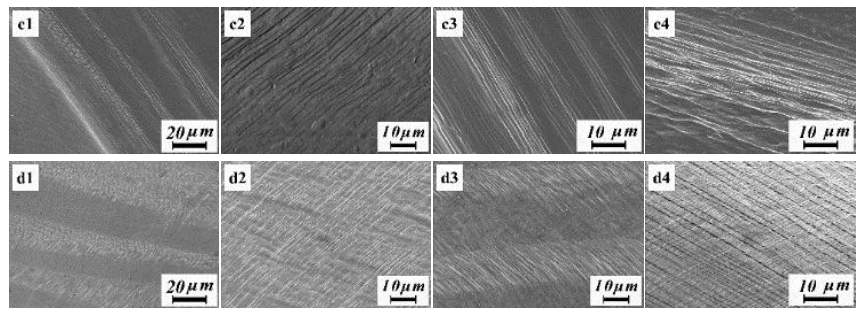

Figure 4: The surface micro-morphology of the single crystal aluminum after dynamic loading: (1) c1, c2, c3 and c4 are the micro-morphology of plane (1-10) after dynamic loading of single crystal aluminum, respectively corresponding to the micrographs of the area indicated by the arrows in Figure 2c; (2) d1, d2, d3 and d4 are micro-morphology of plane (112) after dynamic compression of single crystal aluminum, respectively corresponding to the micrographs of the area indicated by the arrows in Figure 2d

Single crystal aluminum sample was transformed into polycrystal after uniform plastic deformation. In order to study its microstructure evolution, the samples after loading were analyzed by EBSD test technique, and the characteristic pattern of grain orientation and grain boundary distribution was obtained as shown in Figure 6. Figure 6a shows the grain orientation and grain boundary distribution of quasi-statically loaded samples, and Figure 6b shows the grain orientation and grain boundary distribution of the dynamically loaded samples. No twin crystal is observed from the figure, so the deformation mechanism of single crystal aluminum under both quasi-static and dynamic loading is dislocation.

From Figure 6 (a), high-angle, thick strip-shaped deformed structures are observed. There are many low-angle subgrains distributed between adjacent strip-shaped structures. It is speculated that in the process of quasi-static compression, the dislocation density increases with the increase of deformation, forming dislocation walls and dislocation networks which constitute a subgrain boundary with a very small difference in orientation. As shown in Figure 6b, there are differentlyoriented thick strip-shaped deformed structures distributed with a few low-angle subgrains. It is speculated that during dynamic compression, the material deforms rapidly with short dislocation lines concentrated in the local area, forming dislocation wall after entanglement and turns and constituting a subgrain boundary.

Therefore, the microstructure evolution process of single-crystal aluminum from single crystal to polycrystal under quasi-static and dynamic conditions is as follows: during the compression, the singlecrystal aluminum sample firstly forms a large deformation layer via dislocation strip, and then with the increase in deformation, subgrain boundary with a very small orientation difference is formed in the deformed layer. Unstable subgrain boundaries constantly absorb intragranular dislocations during deformation, with dislocation density increased gradually and orientation difference between subgrain boundaries increased. As the orientation difference increases, it changes to a low-angle grain boundary, and then gradually changes into a highangle grain boundary.


Figure 6: Characteristic pattern of grain orientation and grain boundary distribution measured by EBSD: (a) Characteristic pattern of grain orientation and grain boundary distribution of the sample after quasistatic loading (that above $10^{\circ}$ is grain boundary (black), below $3{ }^{\circ}$ is subgrain boundary (blue)) (b) Characteristic pattern of grain orientation and grain boundary distribution of the sample after dynamic loading (that above $10^{\circ}$ is grain boundary (black), below $3{ }^{\circ}$ is subgrain boundary (green))

\section{CONCLUSION}

The deformation mechanisms of single crystal aluminum under quasistatic and dynamic loading are all dislocation. Under the quasi-static loading, the non-uniform plastic deformation process of the single crystal is as follows: due to the slow quasi-static loading process, during the loading, the deformed area first actuates a certain slip system to perform directional plastic deformation by dislocation slip. With the increasing deformation amount, the dislocations continuously accumulate, leading to internal stress concentration, so that another set of slip system is actuated. Then, through the dislocation slip of the set of slip system, the area performs directional plastic deformation. The successively actuated slip systems have different slip directions, and the slip amount is also very likely different. Therefore, the successively actuated slip deformations with different slip ratios result in inhomogeneous macroscopic plastic deformation, and single crystal shape appears as an irregular geometric polyhedron after deformation.

The non-uniform plastic deformation of the single crystal under dynamic loading is as follows: during the loading process, the material deforms rapidly, and a large number of slip systems are actuated almost simultaneously. Dislocations only need a small displacement along each set of slip systems to perform complete deformation, forming a flat and intensive slip band. However, plastic deformation of the material has very obvious local concentration, so single crystal also appears as irregular geometric polyhedron after dynamic loading.

The microstructure evolution of the aluminum single crystal into polycrystal is as follows: during the compression, the single-crystal aluminum sample firstly forms a large deformation layer via dislocation strip, and then with the increase in deformation, the quasi-static sample forms dislocation wall and dislocation network through the internal dislocation accumulation, forming a subgrain boundary with a very small difference in orientation. During dynamic compression, the material deforms rapidly, with short dislocation lines tangled in the local area, forming dislocation wall. Unstable subgrain boundaries constantly absorb intragranular dislocations during deformation, with dislocation density increased gradually and orientation difference between subgrain boundaries increased. As the orientation difference increases, it changes to a low-angle grain boundary, and then gradually changes into a highangle grain boundary.

\section{REFERENCES}

[1] Alfyorova, E.A., Chumaevskii, A.V., Lychagin, D.V., Yu, S., Tarasov. 2015. Macro segmentation and strain hardening stages in copper single crystals under compression. International Journal of Plasticity, 69, 36-53.

[2] Doyama, M., Kogure, Y., Nozaki, T. 2003. Simulation of high speed deformation of copper single crystals. Materials Science and Engineering A, 350, 233-237.

[3] Arakere, N.K., Forest, S., Sabnis, P.A., Yastrebov, V.A. 2013. Crystal plasticity analysis of cylindrical indentation on a Ni-base single crystal super alloy. International Journal of Plasticity, 51, 200-217.

[4] El-Awady, J.A., Tang, Y. 2014. Formation and slip of pyramidal dislocations in hexagonal close-packed magnesium single crystals. Acta Mater, 71, 319-332.

[5] Li, Q. 2013. Microstructure and deformation mechanism of [0001] magnesium single crystal subjected to quasistatic and high-strain-rate compressive loadings. Materials Science and Engineering A, 568, 96-101.

[6] Li, P., Li, S., Wang, Z., Zhang, Z. 2010. Cyclic Deformation Behaviors of [-579]-Oriented Al Single Crystals. Metallurgical and Materials Transactions A, 2532-2537. 